NOTAS 



\title{
LA JUSTICIA CONSTITUCIONAL Y EL DERECHO DE LA UE EN AUSTRIA
}

\author{
FRANZ MERLI ${ }^{1}$ \\ Catedrático de Derecho del Estado y de la Administración \\ Universidad de Viena
}

\begin{abstract}
SUMARIO
I. Introducción. II. El Tribunal Constitucional como Juez de la Unión. III. Subordinación del Tribunal Constitucional al Tribunal de la Unión Europea. IV. El Tribunal Constitucional como defensor de la Constitución. V. El Tribunal Constitucional y la Carta Europea de Derechos Fundamentales. VI. Conclusión.
\end{abstract}

\section{INTRODUCCIÓN}

La posición del Tribunal Constitucional austriaco ante el Derecho de la Unión Europea no es teorética ni principialista sino pragmática y técnica ${ }^{2}$. Los pronunciamientos sobre la naturaleza, la esencia, los principios y la legitimación democrática de la UE o sobre la identidad de la Constitución, los límites de la integración, las facultades para la adopción final de las decisiones son tan escasos en su jurisprudencia como las especulaciones sobre los actos jurídicos de salida de la UE, las propuestas sobre las condiciones de cooperación judicial o la amenaza de las competencias implícitas. Muy al contrario, el Tribunal Constitucional austriaco ha aceptado la primacía del Derecho de la Unión incluso frente al Derecho Constitucional ${ }^{3}$, contribuyendo en gran medida mediante su jurisprudencia a la aplicación del Derecho

1 Versión al castellano de José Ángel Camisón Yagüe, Profesor Contratado Doctor (acreditado por la ANECA a Profesor Titular) de Derecho constitucional en la Universidad de Extremadura

2 Para una visión general del Tribunal Constitucional austriaco, ÖHLINGER T. / PotAcs, M. EU-Recht und staatliches Recht, 5. Aufl. 2014; C. Ranacher/M. Frischbut, Handbuch Anwendung des EU-Rechts, 2. Aufl., 2017. Una comparativa con el modelo alemán puede encontrarse en F. Merli, «Europarechtsfreundlichkeit in der Rechtsprechung des Bundesverfassungsgerichts im Vergleich zum österreichischen Verfassungsgerichtshof,» en: Giegerich (Hrsg.), Der «offene Verfassungsstaat» des Grundgesetzes nach 60 Jabren (2010) 273.

3 V.gr., VfSlg (Repertorio de Jurisprudencia del Tribunal Constitucional) 15.215/1998; 16.050/2000; 17.065/2003; 17.075/2003; 18.642/2008; 19.425 - 19.462/2011. 
de la Unión (vid. II); reconociendo su obligación de subordinación ante el TJUE y observándola (vid. III). Mantiene su posición tradicional de guardián de la Constitución (vid. IV), y para ello no solo continúa siendo un actor fundamental en la creciente aplicación de la Carta de los Derechos Fundamentales, sino también en la protección de los derechos reconocidos en ella (vid. V).

Existe una multiplicidad de razones para esta relación pragmática con el Derecho de la Unión; la principal es, probablemente, el momento y la forma jurídica que adoptó la adhesión de Austria a la Unión Europea. Austria se adhirió a la Unión en 1995, cuando el desarrollo de la integración, que originalmente apenas era predecible, ya se había producido en lo esencial, fundamentalmente, a través de la jurisprudencia del TJUE. Austria conocía así de antemano las implicaciones de su entrada en la UE y, por ello, las cuestiones jurídicas correspondientes ya habían sido intensamente debatidas antes de la adhesión. Algo que fue también un factor decisivo para la forma en la que se produjo la adhesión, que no se condujo como si se tratara de la ratificación de cualquier otro tratado internacional que por su contenido implicara la reforma de la Constitución, sino que se llevó como si de una reforma total de la Constitución se tratara, al amparo de lo previsto el apartado 3 del artículo 44 de la Constitución Federal. La reforma total lleva consigo modificaciones de los principios fundamentales de la Constitución y en este caso, especialmente, de la democracia, de los derechos y de la estatalidad, así como de los pilares en los que la Constitución encuentra su fundamento y, por tanto, solo puede tener lugar si primero existe un significativo respaldo por parte de la mayoría de la ciudadanía mediante referéndum, que en el caso concreto de la adhesión exige de una mayoría de dos tercios del pueblo ${ }^{4}$. La consecuencia de esta técnica es que todos los cambios constitucionales que estuvieron vinculados con la adhesión, por muy profundos que estos fueran, son jurídicamente inatacables 5 . Además, las reformas posteriores de los tratados o sus modificaciones materiales tampoco requieren de referéndum, a menos que estas supongan un cambio sustancial en los principios de la Constitución tal y como fueron fijados tras la reforma de $1995^{6}$, algo que no ha sido en modo alguno el caso después de dicha reforma, es decir, desde el momento

4 La pregunta del referéndum estaba dirigida a consultar sobre la entrada en vigor de una Ley de Reforma Constitucional ( Beitritts-BVG»), mediante la cual se confería la autorización para dar los pasos necesairos para concluir el Tratado de Adhesión (tal y como resultara de la negociación). El texto de la Ley de Adhesión se publicó en Bundesgesetzblatt (BGBl) 1994/744.

$5 \mathrm{Zu}$ den Wirkungen im Einzelnen ÖHLINGer, T,. «EU-BeitrittsBVG», en: Korinek K. / HolouBEK M. (Hrsg.), Österreichisches Bundesverfassungsrecht. Textsammlung und Kommentar (Loseblattsammlung, 1. Lfg., 1999; 12. Lfg. 2016) m.w.N.

6 Según la opinión general este no fue el caso en los Tratados de Ámsterdam, Niza y el Tratado de Adhesión, posteriormente no se materializó el Tratado constitucional y respecto del Tratado de Lisboa hubo distintas opiniones, pero los Estados miembros no lo trataron con carácter general como una reforma constitucional. Desde la reforma constitucional ya no son necesarias nuevas autorizaciones constitucionales especiales, semejantes al modelo del («Beitritts-BVG»), para la aprobación de reformas ordinarias de los Tratados. El reformado artículo 50 de la Constitución Federal requiere para la ratificación de los Tratados de una mayoría de dos tercios del Nationalrat y del Bundesrat, respectivamente. Este artículo ya se utilizó para la ratificación del Tratado de Lisboa. 
de la adhesión y sobre la base del desarrollo que han llevado hasta ahora; eso sí, no será necesario siempre y cuando estas futuras reformas y modificaciones transcurran dentro de lo que es previsible. Aunque seguramente sí pueda preverse, entre otras cosas, una futura labor creadora del Derecho del TJUE. Por lo tanto, el debate fundamental sobre los límites de la transferencia de soberanía y de las transferencias competenciales está, en gran medida, superado ${ }^{7}$. El «autosometimiento» que al parecer algunos Tribunales Constitucionales de otros Estados temen, ya ha tenido lugar en Austria y, además, de un modo constitucionalmente correcto. Que este hecho supone efectivamente la razón fundamental para la contención de la jurisdicción constitucional, lo muestra el contraejemplo del Convenio Europeo de Derechos Humanos. Austria se adhirió al Convenio en 1958. El Parlamento trató inicialmente esta adhesión como un tratado internacional con rango de ley ordinaria, si bien después de los debates de 1964 se le confirió expresamente la correcta categorización de rango constitucional ${ }^{8}$. Aunque en aquel momento no era todavía previsible que la jurisprudencia del Tribunal Europeo de Derechos Humanos pudiera ser tan exhaustiva y creadora de Derecho. Cuando dicha jurisprudencia se estaba desarrollando y, especialmente, la ampliación de la tutela judicial efectiva del artículo 6 del Convenio Europeo de Derechos Humanos, Austria afrontó grandes dificultades, debido a la falta de una jurisdicción contencioso administrativa plena; el Tribunal Constitucional, que, por cierto, ha seguido al Tribunal de Estrasburgo en la mayoría de las ocasiones, no quiso dejar pasar la oportunidad de subrayar que «las contradicciones entre el Ordenamiento Jurídico austriaco y la Convención, según el Estado actual de sus planteamientos, solo pueden ser superadas mediante el resultado de una conformación jurídica abierta a través de las instituciones de la Convención y, por tanto, la pregunta-aqui sin respuesta-que debería ser planteada es si la transferencia de una competencia normativa correspondiente a la esfera constitucional a una organización internacional como la desactivación del <<legislador constitucional >> implicaría una reforma total de la Constitución federal y bubiera requerido de ser sometida a una votación del conjunto del pueblo»9 . El conflicto en ciernes se desactivó entre tanto mediante una reforma de la jurisdicción contencioso administrativa, destacando también, sin duda, la falta de predisposición a la confrontación por parte del Tribunal Constitucional.

\section{EL TRIBUNAL CONSTITUCIONAL COMO JUEZ DE LA UNIÓN}

El Tribunal Constitucional lleva a cabo funciones judiciales de la Unión en diversos ámbitos. En primer lugar, considera las violaciones de la obligación de

7 Aunque los «Actos jurídicos de ruptura» estaban contemplados como una posibilidad en proyecto del ley del Beitritts-BVG, no han jugado ningún papel en la práctica. (1546 der Beilagen zu den Stenographischen Protokollen des Nationalrats, 18. Gesetzgebungsperiode, S. 7).

8 Art. II Z. 7 B-VG-Novelle 1964 BGBl 1964/59.

9 VfSlg 11.500/1987. 
presentar cuestiones prejudiciales por parte de los Tribunales nacionales, según lo establecido en el artículo 267 del TFUE, como violaciones del derecho fundamental estatal al proceso ante el juez ordinario predeterminado por la ley ${ }^{10}$, contribuyendo así a la interpretación uniforme del Derecho de la Unión Europea. Del mismo modo el Tribunal Constitucional sanciona los incumplimientos del Derecho de la Unión como un grave error en la aplicación de la legislación de la Unión (de igual manera que si se tratara de la aplicación del propio derecho nacional), considerándolos como una arbitrariedad o una aplicación «irrazonable» de la ley, y, por lo tanto, los califica como violaciones contra los derechos fundamentales de igualdad o de libertad de la Constitución federal ${ }^{11}$. Algunos ámbitos se refieren, por ejemplo, a las restricciones a la libertad o a las desigualdades de trato basadas en las leyes austriacas, que en virtud del efecto directo de la Unión se tienen, obviamente, por desplazadas y no deberían, por tanto, haber sido aplicadas. De este modo asegura, con carácter general, la primacía del Derecho de la Unión y, concretamente, la inaplicación de la legislación nacional contraria a la Unión por parte de las autoridades administrativas, quienes a menudo necesitan un acicate en este sentido para ello.

De igual forma se aplica respecto de la específica petición de revisión judicial: no se permiten nuevos cuestionamientos por parte de los Tribunales ni otras actuaciones como la iniciación de oficio de procedimientos ante el Tribunal Constitucional cuando el desplazamiento de la norma correspondiente resulta evidente en virtud de la primacía del derecho de la Unión (bien apriorísticamente o bien en virtud del correspondiente pronunciamiento del Tribunal de Justicia de la UE); en ese caso el Tribunal Constitucional considera que no hay entonces siquiera cuestión alguna que analizar ${ }^{12}$. Para que se produzca la admisibilidad de las llamadas peticiones individuales de revisión judicial (en terminología alemana: cuestiones de constitucionalidad contra leyes y otras normas de alcance general) debe constatarse, no obstante, de forma concluyente que la norma controvertida no pueda ser efectivamente desplazada, dado que ello conllevaría la

10 V. gr. VfSlg 14.390/1995; 14.607/1996; 14.889/1997; 15.139/1998; 15.507/1999; 15.657/1999; $15.766 / 2000 ; 15.810 / 2000 ; 16.391 / 2001 ; 16.757 / 2002 ; 16.988 / 2003 ; 17.411 / 2004 ; 17.500 / 2005$; 18.541/2008; 18.803/2009; 18.834/2009; 19.118/2010; VfGH B 1537/2012 vom 7.6.2013.

Era obligatorio que los órganos administrativos fueran independientes a semejanza de los tribunales, y contra sus decisiones no cabía apelación ante el Tribunal contencioso administrativo, por lo que eran tenidos por tribunales de última instancia, como lo previsto en el art. 267 del TFUE. Desde la reforma del orden contencioso administrativo, que entró en vigor en 2014, los Tribunales contencioso administrativo siempre deciden antes que el Tribunal Constitucional; desde entonces, por norma general, las decisiones administrativas pueden ser recurridas ante los Tribunales contencioso administrativos. El Tribunal Contencioso administrativo tiene solo obligación de consultar si puede existir una violación que afecte al Tribunal Constitucional.

11 V. gr. VfSlg 14.886/1997; 15.448/1999; 15.815/2000; 15.450/2001; 15.910/2000 [añadir el prefijo E desde 2001]; 16.771/2002; 17.614/2005; 18.414/2008; 18.555/2008; 18.618/2008; 19.628/2012; $19.661 / 2012$.

12 V. gr. VfSlg 15.368/1998; 16.995/2003; 18.298/2007; $19.425-19.462 / 2011 ; 19.495 / 2011 ;$ 19.568/2011; VfGH B 1537/12 de 7.6.2013. 
posibilidad de lesiones en el derecho del demandante ${ }^{13}$, en ese caso el Tribunal Constitucional realiza una «verificación exhaustiva» de la conformidad del Derecho de la Unión con la norma controvertida. En todos estos casos el conflicto jurídico constitucional tiene lugar no solo en el nivel de la admisibilidad, sino que a través del rechazo de solicitudes de revisión judicial por cuestiones de admisibilidad se asegura en definitiva la aplicación efectiva del Derecho de la Unión al litigio principal, y también la obligación que los órganos judiciales y los tribunales contencioso administrativos tienen de no aplicar el derecho nacional contrario al Derecho de la Unión. Por su parte, en el ámbito del control abstracto de las normas, los asuntos de desplazamiento en virtud del Derecho de la Unión no juegan ningún papel ${ }^{14}$.

En lo que se refiere al estudio sustantivo de controversias jurídicas, en principio, no se tratan cuestiones relativas al Derecho de la Unión; así el único parámetro de validez es el Derecho constitucional, con una única y singular excepción, pues por debajo de la Constitución el Tribunal Constitucional toma en consideración también, en su caso, el contexto del Derecho de la Unión Europea; en tanto que las especificidades del Derecho de la Unión Europea pueden constituir el interés público, servir de base a los derechos fundamentales, o llegar a ofrecer un fundamento objetivo para justificar un trato diferenciado ${ }^{15}$. El Tribunal considera que en los casos de control de constitucionalidad de las leyes no está obligado a interpretar la conformidad de las normas examinadas con el Derecho de la Unión ${ }^{16}$ para no generar así situaciones contrarias al Derecho de la Unión ${ }^{17}$. Ello da lugar a pensar que el parámetro de validez — la Constitución — podría verse desplazada por el Derecho originario de la Unión. La confirmación de ello se puede encontrar en otros procesos ante el Tribunal Constitucional. Así, por ejemplo, el Tribunal Constitucional declaró inaplicables los límites constitucionales de los controles contencioso administrativos del Regulador de las Telecomunicaciones por ser contrarios a una importante directiva ${ }^{18}$. Otro ejemplo ilustrativo es el caso de la publicación de retribuciones de los funcionarios de las empresas públicas; al que siguieron otros más ${ }^{19}$.

Finalmente, el Tribunal Constitucional actúa como tribunal de la Unión fallando sobre reclamaciones de responsabilidad patrimonial derivada de incumplimientos judiciales o legislativos del Derecho de la Unión Europea ${ }^{20}$. Las

13 V. gr. VfSlg 15.771/2000; 18.298/2007; 19.495/2011.

14 V. gr. VfSlg 15.753/2000.

15 V. gr. VfSlg 14.075/1995; 16.764/2002; 17.672/2005; VfGH G 450/15 ua de 2.7.2016.

16 V. gr. VfSlg 14.391/1995; 16.737/2002; 19.518/2011; 19.591/2011; 19.890/2014.

17 V. gr. VfSlg 16.027/2000; 18.642/2008; VfGH G 25/16 ua de 15.6.2016.

18 VfSlg 15.427/1999.

19 VfSlg 17.065/2003; infra nota 28.

20 V. gr. VfSlg $16.107 / 2001 ; 17.002 / 2003 ; 17.019 / 2003 ; 17.095 / 2003 ; 17.214 / 2004 ; 17.576 / 2005$; 18.409/2008; 18.448/2008; 18.557/2008; 19.361/2011; 19.428/2011; 19.757/2013; VfGH A 3/15 de 11.06.2015. 
normas austriacas de responsabilidad patrimonial no contemplan este tipo de reclamaciones que, por lo tanto, no pueden ser invocadas ante los tribunales ordinarios al no ser estos los tribunales ordinarios de responsabilidad patrimonial. El Tribunal Constitucional suple esta laguna en base a una competencia general subsidiaria prevista en el artículo 137 de la Constitución Federal para casos de reclamaciones patrimoniales contra los poderes públicos.

\section{SUBORDINACIÓN DEL TRIBUNAL CONSTITUCIONAL AL TRIBUNAL DE LA UNIÓN EUROPEA}

El Tribunal Constitucional reconoce su propia obligación de subordinación ante el Tribunal de la Unión Europea, y en conjunto la observa ${ }^{21}$. Hasta el momento ha iniciado cuatro cuestiones prejudiciales ante el Tribunal de Justicia de la Unión Europea sobre la interpretación y/o la validez del Derecho de la Unión, y en ellas queda bien ilustrado lo dicho hasta ahora.

En el primer caso se discutía si la devolución de un determinado impuesto era o no una ayuda de Estado. El Tribunal Constitucional consultó así si la devolución, que la ley preveía solo para una parte de las empresas afectadas, podía ser constitutiva de una ayuda; preguntó no solo teniendo en cuenta las diversas opciones que podría tener su decisión en este caso, si no también sobre si cualquier devolución a empresas podría ser considerada una ayuda ${ }^{22}$. Como cabía de esperar, el Tribunal de Justicia respondió afirmativamente a la primera pregunta y contestó negativamente a la segunda ${ }^{23}$. En base a esta respuesta pudo el Tribunal Constitucional emitir su resolución al caso $^{24}$. Entendió así que la limitación de la devolución a unas empresas determinadas no debía ser aplicada por los poderes públicos, porque ello daba lugar a una ayuda no notificada y, por tanto, contraria al actual apartado 3 del artículo 108 del TFUE. Este fue un caso en el que se constató una arbitrariedad administrativa (aunque solo reconocible tras las sentencias del Tribunal de Justicia de la Unión, pero en todo caso relevante ${ }^{25}$, y el Tribunal Constitucional anuló la correspondiente decisión de las Autoridades Públicas. Por lo tanto, nos encontramos aquí con un ejemplo de un caso en el que una ley fue anulada porque la misma no era aplicable de conformidad con lo establecido en los fundamentos del Derecho de la Unión ${ }^{26}$.

El Tribunal Constitucional no se subordina a las prescripciones del Derecho de la Unión Europea de forma impotente, sino que, como muestra el

21 V. gr. VfSlg 15.450/1999; 16.627/2002; 18.797/2009; 19.702/2012.

22 VfSlg 15.450/1999.

23 EuGH Rs. C-143/99, Adria-Wien Pipeline u.a., Slg. 2001 I-8384.

24 VfSlg 16.401/2001.

25 Vid. supra. nota 10.

26 Vid. supra. nota 11. 
segundo caso, puede utilizar dicha subordinación de modo creativo. Cuando el Tribunal Constitucional tuvo que evaluar la legalidad de la publicación de los ingresos de ciertos altos funcionarios del ente público de radiodifusión, el Constitucional tuvo que considerar, de una parte, el derecho fundamental a la vida privada del artículo 8 del CEDH y, de otra parte, una disposición específica de una ley que limitaba las remuneraciones de los funcionarios públicos, disposición esta que también desempeña un papel constitucional y en la que se preveía dicha publicación bajo determinadas circunstancias. El Constitucional se encontraba, por tanto, ante un conflicto normativo de nivel constitucional. $\mathrm{Y}$, en tanto que no se mostraba partidario de una solución favorable a la obligación de publicar (que era la que correspondía en virtud de la aplicación de los principios de «lex posterior» y/o «lex specialis»), optó por elevar una cuestión prejudicial al TJUE sobre el efecto directo y el contenido de la legislación europea sobre protección de datos ${ }^{27}$. El TJUE afirmó que la directiva gozaba de efecto directo y, en el marco del artículo 8 del CEDH y de la jurisprudencia del Tribunal Europeo de Derechos Humanos; por otra parte, señaló que la directiva amparaba la publicación «si a la vista del objetivo perseguido por la norma constitucional se lograba una administración pública apropiada de los fondos públicos», circunstancia esta que, sin embargo, debía ser apreciada en su caso por el Tribunal que había formulado la cuestión ${ }^{28}$. Por lo que al contenido se refiere el TJUE devolvió la cuestión al Tribunal Constitucional. Esto negaba la necesidad de intervención y en base a ello se podían considerar como desplazadas las disposiciones constitucionales de limitación de la remuneración en base a la primacía de la directiva de protección de datos ${ }^{29}$. Independientemente de cuales sean los elementos del razonamiento, cabe destacar en todo caso, que se fortalece la primacía del Derecho de la Unión Europea incluso frente a las normas de rango constitucional ${ }^{30}$ como la Constitución ${ }^{31}$ o el Convenio Europeo de Derechos Humanos por el sinuoso camino de la aplicación normativa de la Unión Europea.

El punto de partida para la tercera cuestión prejudicial estuvo en la impugnación de las elecciones a un importante órgano de la Cámara del Trabajo (órgano público austriaco de representación de los trabajadores), en las cuales se había removido de la lista de candidatos a los trabajadores turcos porque, según la ley, solo los ciudadanos austriacos podían ser candidatos. En respuesta a tan significativa cuestión del Tribunal Constitucional ${ }^{32}$, el TJUE sentenció que la Decisión 1/80 de Consejo de Asociación entre la Comunidad Europea y

27 VfSlg. 16.050/2000.

28 EuGH Rs. C-465/00, Österreichischer Rundfunk u.a., Slg. 2003 I-4989.

29 VfSlg 17.065/2003.

30 Vid. supra. FN 7.

31 Vid. supra. FN 2.

32 VfSlg 16.100/2001. 
Turquía, que confería a los trabajadores turcos las mismas «condiciones de trabajo» era directamente aplicable e impedía, por tanto, la exclusión de su derecho de sufragio pasivo ${ }^{33}$. En virtud de ello, el Tribunal Constitucional suspendió aquellas elecciones y obligó a repetirlas permitiendo participar en ellas a los candidatos turcos.

La cuarta cuestión prejudicial condujo en última instancia a la anulación de la normativa sobre conservación de datos personales. En lo concerniente al litigio principal, varios miles de austríacos interpusieron ante el Tribunal Constitucional una petición de derogación de las disposiciones legales adoptadas en Austria para la transposición de la Directiva 2006/24 relativa a la conservación de datos ${ }^{34}$. En esta ocasión el Tribunal Constitucional consultó no solo sobre la interpretación de una Derecho de la Unión Europea (concretamente el art. 8 de la Carta Europea de Derechos Fundamentales), sino también sobre la validez de significativas disposiciones de la Directiva ${ }^{35}$. El TJUE declaró, como se sabe, la nulidad de la Directiva, en tanto que la misma violaba los artículos 7, 8 y 52 de la Carta $^{36}$. En este caso no había legislación de la Unión, que hubiera podido desplazar a la normativa nacional (incluida la constitucional ${ }^{37}$ o que hubiera podido hacer inadmisible su aplicación ${ }^{38}$; de modo que el Tribunal Constitucional austriaco examinó las disposiciones sobre la conservación de datos tomando como parámetro el Derecho Constitucional austriaco y derogó dichas disposiciones en tanto que constitutivas de una injerencia desproporcionada de los derechos fundamentales a la protección de datos ( $\$ 1$ de la Ley de Protección de datos) y a la vida familiar (art. 8 $\mathrm{CEDH})^{39}$.

Queda por señalar que el Tribunal Constitucional era el órgano judicial que resolvía en primera y única instancia respecto de estos asuntos concretos, y es por ello que fue él a quien le correspondió presentar la correspondiente cuestión prejudicial. Esto continúa siendo así en aquellos procedimientos en los que se cuestionan leyes, pero desde la reforma de la jurisdicción contencioso administrativa que entró en vigor en el año 2014, los Tribunales contencioso administrativos son quienes deciden en primera instancia sobre los actos administrativos y, por ello, están legitimados para plantear por sí mismos cuestiones prejudiciales ${ }^{40}$, evitando así la necesidad de que estas se formulen por el Tribunal Constitucional.

33 EuGH Rs. C-171/01, Wählergruppe Gemeinsam, Slg. 2003 I-4301.

34 VfSlg 19.702/2012.

35 VfSlg 19.892/2014.

36 EuGH verb. Rs. C-293/12 und C-594/12, Digital Rights Ireland und Kärntner Landesregierung u.a., Slg. 2014 I-0000.

37 Vid. supra. nota 2.

38 Vid. supra. nota 12.

39 VfSlg 19.892/2014.

40 Vid. también supra nota 9. 


\section{EL TRIBUNAL CONSTITUCIONAL COMO DEFENSOR DE LA CONSTITUCIÓN}

Evidentemente la Corte Constitucional austriaca es, fundamentalmente, un Tribunal Constitucional y no solo un Tribunal de la Unión Europea, por ello en su actuación defiende la Constitución también contra el Derecho de la Unión, principalmente respecto de la transposición del Derecho de la Unión, como muestra su decisión sobre la conservación de datos personales. Para ello ha desarrollado un principio de vinculación dual hacia el Derecho de la Unión Europea y la Constitución, si bien en la práctica se ocupa, principalmente, de asuntos relativos a la organización del Estado y del proceso legislativo ${ }^{41}$. Así, mientras que el compromiso dual — que es en realidad un compromiso singular a falta de legislación europea - aparece como evidente respecto de la implementación del Derecho de la Unión, y también en lo relativo a la competencia, el procedimiento y los requisitos formales de la legislación sobre reserva de Ley y principio de legalidad; en lo relativo a la normativa nacional que contenga normativa de la Unión europea este principio está dando lugar a significativos problemas. La jurisprudencia no es uniforme en este punto. Así el Tribunal Constitucional no ha seguido el principio de la vinculación dual en lo relativo al establecimiento por vía legislativa de ciertas autoridades independientes encargadas de la supervisión de la asignación de contratos públicos, debido a que éstas son contrarias a los principios constitucionales de autonomía municipal y a la posición jerárquicamente superior de ciertos órganos administrativos, siendo por ello inconstitucionales; aunque fuera necesario su establecimiento de conformidad con la normativa europea, la solución a la controversia solo podía ser alcanzada mediante una reforma constitucional $^{42}$. Por el contrario, el Tribunal Constitucional ha desplazado en otros casos el precepto constitucional controvertido ${ }^{43}$.

\section{EL TRIBUNAL CONSTITUCIONAL Y LA CARTA EUROPEA DE DERECHOS FUNDAMENTALES}

Por muy importante que sea la Corte Constitucional austriaca su significación como «tribunal constitucional» y como Tribunal de la Unión es cada vez menor en el marco del progresivo proceso de europeización. De una parte, cada vez más cuestiones se encuentran reguladas dentro del ámbito del Derecho de la Unión y, por lo tanto, están dotadas de primacía, incluso frente al Derecho

41 V. gr. VfSlg 15.189/1998; 17.022/2003; 17.967/2006.

42 VfSlg 17.001/2003; 15.578/1999; 16.002/2000.

43 VfSlg 15.427/1999. Vid. también las observaciones en el citado caso de la conservación de datos, vid. supra nota 36. 
constitucional estatal; por otra parte, cada vez más situaciones están dentro del ámbito de competencias del Derecho de la Unión Europea y por ello también de la Carta de Derechos Fundamentales. Sin embargo, el Tribunal Constitucional no puede pronunciarse sobre su interpretación en tanto que - como ocurre con todo el Derecho de la Unión- ${ }^{44}$ esta no se considera como una norma constitucional austriaca. El diálogo lo llevan otros tribunales y no el Tribunal Constitucional. Así, cada tribunal e, incluso, cada autoridad administrativa puede inaplicar la normativa estatal que sea contraria a la Carta. Con ello el Tribunal Constitucional ha perdido el monopolio del control normativo tanto en el ámbito de los derechos fundamentales como en la interpretación de la soberanía.

Este es el contexto para una sentencia ${ }^{45}$ del Tribunal Constitucional en la que equiparó los derechos de la Carta Europea a los derechos constitucionalmente garantizados por la Constitución. Así la Carta, cuya formulación y clasificación de derechos es semejante a los derechos garantizados por la Constitución, pasó a ser para el Tribunal Constitucional un instrumento de control de los actos administrativos y las leyes, a diferencia del resto del Derecho de la Unión. Este reconocimiento vino de la mano de dos quejas de demandantes de asilo que alegaban una infracción por parte del Tribunal de Asilo del derecho al juez ordinario predeterminado por la ley al amparo de lo establecido en el artículo 47 de la Carta. El Tribunal constitucional consideró el caso como admisible en base a los principios del Derecho de la Unión, en concreto en virtud de la obligación de asegurar una protección jurídica equivalente a para los derechos estatales y los de la Unión Europea, por lo tanto, el Tribunal consideró obligatorio tratar el derecho previsto en el artículo 47 de la Carta como un derecho constitucionalmente garantizado. Sin embargo, por lo que respecta al fondo el Tribunal desestimó finalmente ambas quejas.

Esta sentencia ha recibido muchas críticas ${ }^{46}$. De hecho, dogmáticamente tiene una difícil justificación. El principio de protección jurídica equivalente no exige la equiparación entre los derechos de la Carta y los derechos constitucionalmente garantizados; algo que también ha sido confirmado por el TJUE, y no en base a una consulta del Tribunal constitucional, sino que quien lo consultó primeramente fue el Tribunal Supremo, que es la otra Alta Corte de Justicia austriaca $^{47}$. La equiparación pueden sonar bien pero genera algunas dificultades porque en el modelo descentralizado de control normativo del Derecho de la

44 Que ello no constituye para él un parámetro de validez, lo había dicho ya en repetidas ocasiones el propio Tribunal Constitucional: v. gr. VfSlg. 15.753/2000, 15.810/2000.

45 VfSlg 19.632/2012.

46 V. gr. MAYR, S. Verfassungsgerichtlicher Prüfungsgegenstand und Prüfungsmaßstab im Spannungsfeld nationaler, konventions- und unionsrechtlicher Grundrechtsgewährleistungen, ZfV 2012, 401; MERLI, F. Umleitung der Rechtsgeschichte, JRP 2012, 335; PösCHL, M. Verfassungsgerichtsbarkeit nach Lissabon, ZÖR 2012, 587.

47 EuGH Rs. C-122/13, A gegen B u.a., Slg. 2014 I-2195. El proceso no era relativo a la coincidencia de preceptos legales de la Unión, pero el Tribunal admitió el establecimiento de un control ordinario del Derecho de la Unión, que el TJUE acepta solo bajo ciertas restricciones. 
Unión, en el cual todas las autoridades y jueces pueden también inaplicar las leyes contrarias al Derecho de la Unión en determinados casos, difícilmente casa con el modelo austriaco, en el cual todas las autoridades y jueces pueden también inaplicar una ley contraria a la Constitución pero solo en tanto en cuanto ésta haya sido derogada previamente por el Tribunal Constitucional. Y, por último, cabe señalar que el Tribunal Constitucional en su decisión se reconoció a sí mismo derechos que solo podían haberle sido reconocidos por el «legislador constitucional» pero olvidó, no obstante, proteger los derechos de los demandantes de amparo $^{48}$.

En todo caso la sentencia es en cierto modo compresible, porque esta asegura asimismo también al Tribunal Constitucional su participación dentro del ordenamiento constitucional europeo. Podría pensarse que el Tribunal Constitucional quiere ser un importante y privilegiado interlocutor del TJUE frente a otras instancias austriacas de garantía de los derechos, que filtra los casos austriacos, los clarifica, si es necesario, y, en su caso, se los remite al TJUE a través de una cuestión prejudicial de la que extrae después las consecuencias apropiadas para el ordenamiento jurídico y también de naturaleza general. Probablemente se considera a sí mismo junto con los Tribunales Constitucionales de otros Estados miembros como un catalizador especialmente adecuado para el futuro desarrollo de la garantía de los derechos fundamentales en la Unión Europea y, por lo tanto, como una parte del particular sistema europeo de garantía de los Derechos fundamentales.

Pero este no es el caso, porque el Tribunal Constitucional austriaco apenas ha formulado al TJUE cuestiones prejudiciales sobre la Carta. Como se mencionó antes adoptó, incluso, aquella sentencia, sin cuestión prejudicial previa ${ }^{49}$, en tanto que el Tribunal Constitucional, que es en ciertas circunstancias reacio a plantear cuestiones, sostiene que no corresponde su interposición cuando la cuestión susceptible de consulta, a la luz del CEDH y de la jurisprudencia del TEDH y de otros Altos Tribunales, no vaya a ser decisiva, o bien cuando el caso pueda ser resuelto tomando como referencia un derecho constitucional protegido con el mismo ámbito de aplicación, especialmente si se trata de un derecho contenido en el CEDH.

A este respecto el Tribunal Constitucional ha resuelto, sin plantear cuestión prejudicial alguna, respecto de la constitucionalidad de una nueva cláusula del derecho de asilo del art. 47 de la Carta solo mediante una cita a la jurisprudencia

48 Esto lo ha hecho mejor en otras sentencias posteriores sobre el mismo principio, pero en este caso se concentró en el demandante.V. gr. VfGH U 1175/12 ua de 13.3.2013.

49 En un proceso posterior un demandante solicitó la responsabilidad del Estado por la violación que suponía someterse al Tribunal Constitucional. El Tribunal Constitucional, que debía decidir sobre sí mismo, estableció que la violación de la obligación de someterse a él no solo no fundamenta ninguna responsabilidad del Estado, sino que además señaló que el demandante no había demostrado una violación flagrante del Derecho de la Unión, y desestimó la demanda. VfSlg 19.757/2013. 
del $\mathrm{TEDH}^{50}$, mientras que en otros casos ha recurrido a las garantías previstas en la Carta; todo ello a pesar de la posibilidad que tiene poder recurrir a la aplicación de los derechos fundamentales austriacos (que incluyen los previstos en el CEDH) -así lo hizo en los casos de discriminación por motivos de orientación sexual ${ }^{51}$, del derecho al juez ordinario predeterminado por la $l e y^{52}$, el derecho a la propiedad o también los derechos a la propiedad privada ${ }^{53}$ y a la protección de datos tras la anulación de la directiva de protección de datos, por el Tribunal de Justicia de la $\mathrm{UE}^{54}$.

\section{CONCLUSIÓN}

En general, el Tribunal Constitucional alemán se toma muy en serio el Derecho de la Unión Europea. No solo es que, por principio, no se oponga al mismo por, sino que, además, contribuye a su aplicación. Por otra parte, mediante una jurisprudencia diferenciada ha encontrado un enfoque constructivo que, sin descuidar su propio papel, sirve también al Derecho Constitucional austriaco.

$$
* * *
$$

TitLe: Constitutional Justice and EU Law in Austria

ABSTRACT: This paper analyses the role of the Austrian Constitutional Court in relation to the application of EU law. It focuses on the European dimension of the main Constitutional Court rulings on EU matters and shows the Constitutional Court of Austria as an European Union Court.

Resumen: Este trabajo analiza el papel de Tribunal Constitucional austriaco en relación con la aplicación del Derecho de la UE. Se centra en la dimensión europea de los principales pronunciamientos de la Corte Constitucional sobre asuntos relativos a la UE y muestra a la Corte Constitucional de Austria como un Tribunal de la Unión Europea.

KeY Words: European Union, Austria, Austrian Constitutional Court, Jurisprudence.

Palabras Clave: Unión Europea, Austria, Tribunal Constitucional austriaco, Jurisprudencia.

FECHA DE RECEPCIÓN: 15.02.2017

FeCHA DE ACEPTACIÓN: 28.02.2017

50 VfSlg 19.790/2013.

51 VfSlg 19.865/2014.

52 VfSlg 19.916/2014.

53 VfSlg 20.000/2015.

54 VfSlg 19.892/2014; sobre ello vid. supra nota 38. En lo relativo a las cuestiones prejudiciales (nota 34), el Tribunal Constitucional solo ha presentado una cuestión prejudicial interpretativa relativa a la Carta, que resultó irrelevante por la derogación por el TJUE de la directiva (nota 35). 